\title{
Percepción sobre la Cultura Emprendedora en la facultad de Ciencias Administrativas de la UNMSM
}

\author{
The Entrepreneurial Culture at The Faculty of Administrative \\ Sciences of The UNMSM
}

\begin{abstract}
RESUMEN
El presente estudio describe la percepción de la cultura emprendedora en la Facultad de Ciencias Administrativas (FCA) de la Universidad Nacional Mayor de San Marcos (UNMSM), En donde la población de estudio fueron los estudiantes y docentes de la facultad, a quienes se les aplicó encuestas durante el semestre académico 2016 II. A partir de los hallazgos de la investigación, se proponen reflexiones de interés para la gestión y el gobierno en las universidades, que promueven competencias de emprendimiento. Los problemas de investigación fueron ¿Existe una cultura emprendedora en la Facultad de Ciencias Administrativas (FCA) de la UNMSM?, y ¿Esta cultura emprendedora, es fortalecida con actividades y clima organizacional, que motive el desarrollo del emprendimiento? Uno de los principales resultados obtenidos fue que los estudiantes perciben que hay "regular fomento" de la cultura emprendedora con un 53\%; en tanto en los docentes "poco fomento" en un 50\%. Por tal motivo, ambos grupos consideran que la FCA "deben intensificar el fomento de la cultura emprendedora", en estudiantes con un $92 \%$ y docentes con un $87 \%$.
\end{abstract}

Palabras claves: Cultura emprendedora; innovación; desarrollo organizacional.

\begin{abstract}
The present study describes the perception of the entrepreneurial culture in the Faculty of Administrative Sciences (FCA) of the National University of San Marcos (UNMSM), the study population were students and teachers of the faculty, who were surveyed During the academic semester 2016 II. Based on the research findings, reflections are proposed that promote entrepreneurship competencies of interest for management and government in universities. The research problems were: Is there an entrepreneurial culture in the Faculty of Administrative Sciences (FCA) of the UNMSM? And Is this entrepreneurial culture strengthened by activities and organizational climate that motivates the development of entrepreneurship? One of the main results obtained was that students perceive that there is "regular promotion" of the entrepreneurial culture with 53\%; while in teachers "little development" by $50 \%$. For this reason, both groups consider that the FCA "should intensify the promotion of the entrepreneurial culture", in students with $92 \%$ and teachers with $87 \%$.
\end{abstract}

Keywords: Entrepreneurial culture; innovation; organizational development.
Emma Emília Pérez Palácios $^{1}$

eeperezpalacios@gmail.com

Pedro Leonardo Tito Huamaní

pedrotito18@hotmail.com

\section{Graciela Thupa Velásquez ${ }^{2}$}

gratv20@gmail.com

Universidad Nacional Mayor de San Marcos, Facultad de Ciencias Administrativas

1 Universidad Nacional Mayor de San Marcos

2 Universidad Nacional Mayor de San Marcos, Facultad de Ciencias Administrativas, Centro de investigación. Lima, Perú.

(C) Los autores. Este artículo es publicado por Gestión en el Tercer Milenio de la Facultad de Ciencias Administrativas de la Universidad Nacional Mayor de San Marcos. Este es un artículo de acceso abierto, distribuido bajo los términos de la licencia Creative Commons Atribucion - No Comercia_Compartir Igual 4.0 Internacional. (http://creativecommons.org/licenses/by-nc-sa/4.0/) que permite el uso no comercial, distribución y reproducción en cualquier medio, siempre que la obra original sea debidamente citada. 


\section{INTRODUCCIÓN}

El presente estudio pretende visibilizar el estado actual de la cultura emprendedora en la Facultad de Ciencias Administrativas (FCA) de la Universidad Nacional Mayor de San Marcos (UNMSM), con el propósito de facilitar a los gestores y decisores institucionales, para que tomen medidas correctivas, y fomenten la cultura emprendedora entre los estudiantes y docentes.

El fomento de la cultura emprendedora y la innovación son acciones claves para el pleno desarrollo de la tercera misión de la universidad: Formar en emprendimiento y establecer la relación empresa-universidad (Campos, Hormiga, \& Valls, 2014). Consciente de ello, se analizaron los esfuerzos de la FCA de la UNMSM para apoyar iniciativas emprendedoras y/o innovadoras y la percepción de la comunidad universitaria, respecto de ello.

Podemos considerar que la primera idea de emprender un negocio propio, nace cuando los individuos sienten que sus esfuerzos en el trabajo no son suficientemente recompensados, o cuando pasan a formar parte del grueso de desempleados y subempleados en el país. El desempleo ha causado, que más personas opten por crear sus propias fuentes de ingreso, siendo a este nivel una economía más de subsistencia. Posteriormente, ya consolidado el proyecto emprendedor, es natural que dicha idea plasmada mejore su calidad de vida. Desde sus experiencias vividas como dependiente, es posible que consideren tener los contactos suficientes y los recursos financieros necesarios para lanzarse a la actividad empresarial, pues esto supone tener mayor libertad y seguridad, obtener prestigio al convertirse en propietario de una empresa y tener control sobre las horas a trabajar, con que intensidad trabajar, a quién contratar, entre otros. Sin embargo, a pesar de que tales son ventajas significativas, en el emprendedor suelen presentarse obstáculos de índole administrativos y financieros fundamentalmente, acceso a fuentes de información privilegiada, extrema burocracia en organismos públicos ligados a la promoción emprendedora, entro otros (Perez, Sumari, Quintana, \& Uyehara, 2013).

Desde el punto de vista psicológico, llevar adelante un emprendimiento propio requiere una amplia gama de habilidades y un conjun- to de atributos que configuren una personalidad emprendedora. Antes de lanzarse a la actividad empresarial, en condiciones ideales se recomienda asegurarse contar con un perfil emprendedor. De no poseerlo, los seres humanos podemos aprender, incorporando conductas y actitudes, que con el correr del tiempo podemos desarrollarlas. Es este último la que motiva el presente estudio.

Visto así, en tiempos de competitividad, el papel de las universidades no solo se restringe a las actividades de enseñanza y trasmisión de conocimientos, de investigación; sino, ahora la sociedad demanda una mayor implicación de las universidades en el desarrollo económico y social de la sociedad, región y país. Este proceso ha originado la aparición y consolidación de un nuevo tipo de universidades: Universidad Emprendedora.

El emprendimiento ${ }^{1}$ es un tema actual por factores como la globalización, economía y educación (Narváez, 2012) y está ligado a la cultura emprendedora, que se relaciona con la iniciativa y acción (Eoi, 2017). Para este último la cultura emprendedora se relaciona con:

- El conocimiento para lanzar nuevos proyectos propios con autonomía, con capacidad de asumir riesgo, responsabilidad, intuición, resolución de problemas, proyección al exterior y de reacción.

- El conocimiento para llevar a cabo proyectos de otros con el mismo espíritu de innovación, responsabilidad y autonomía.

En referencia a lo anterior, la cultura emprendedora alienta y anima a los individuos para promulgar sus ideas y concretarlas. Lo afirmado se puede corroborar en el estudio aplicado a una muestra de emprendedores y empresas brasileras del Distrito Federal de Brasil (Eda \& Cristina, 2007), donde los resultados de la investigación confirman la relación entre cultura emprendedora y la actitud. Esto se explica, cuando se entiende los valores como la capa más profunda de la cultura, demostrando una relación directa con la percepción que

1 Emprendimiento: Acción y efecto de emprender (acometer una obra). Cualidad de emprendedor. 
tienen las personas sobre el medio ambiente y como lo relacionan con la forma de pensar, sentir y de comportarse. Como vemos, la actitud emprendedora está vinculada a dimensiones culturales, psicológicas y tecnológicas.

Dentro de este contexto existen factores que estimulan el espíritu emprendedor, y estos son cuatro principalmente como se aprecia en la figura 1 (Narváez, 2012). Tales factores afectan en la creación y el desarrollo de universidades emprendedoras (Guerrero, 2006) y (Sooreh, Salamzadeh, \& Safarzadeh, 2011).

En ese orden de ideas, siendo la educación el elemento que influye en la configuración del perfil emprendedor, el papel de las universidades emprendedoras se convierten en un factor determinante para promover competencias emprendedoras entre sus estudiantes.

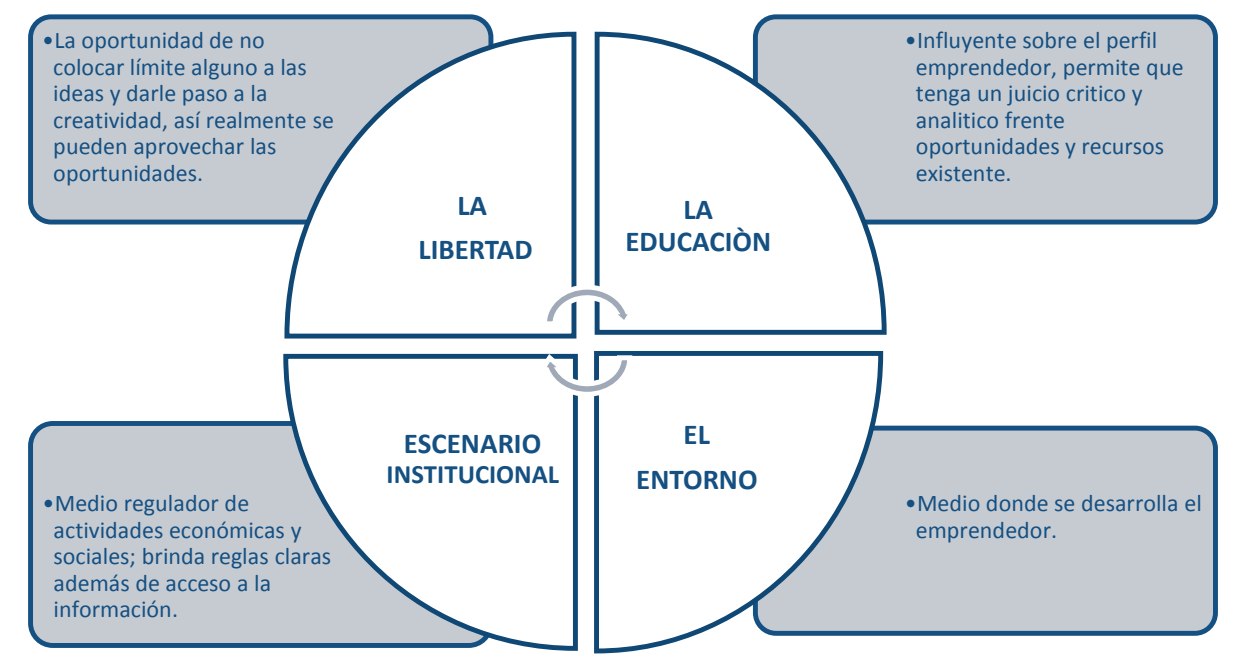

Figura 1. Factores que influyen en el espíritu emprendedor Elaboración propia. Fuente (Narváez, 2012)

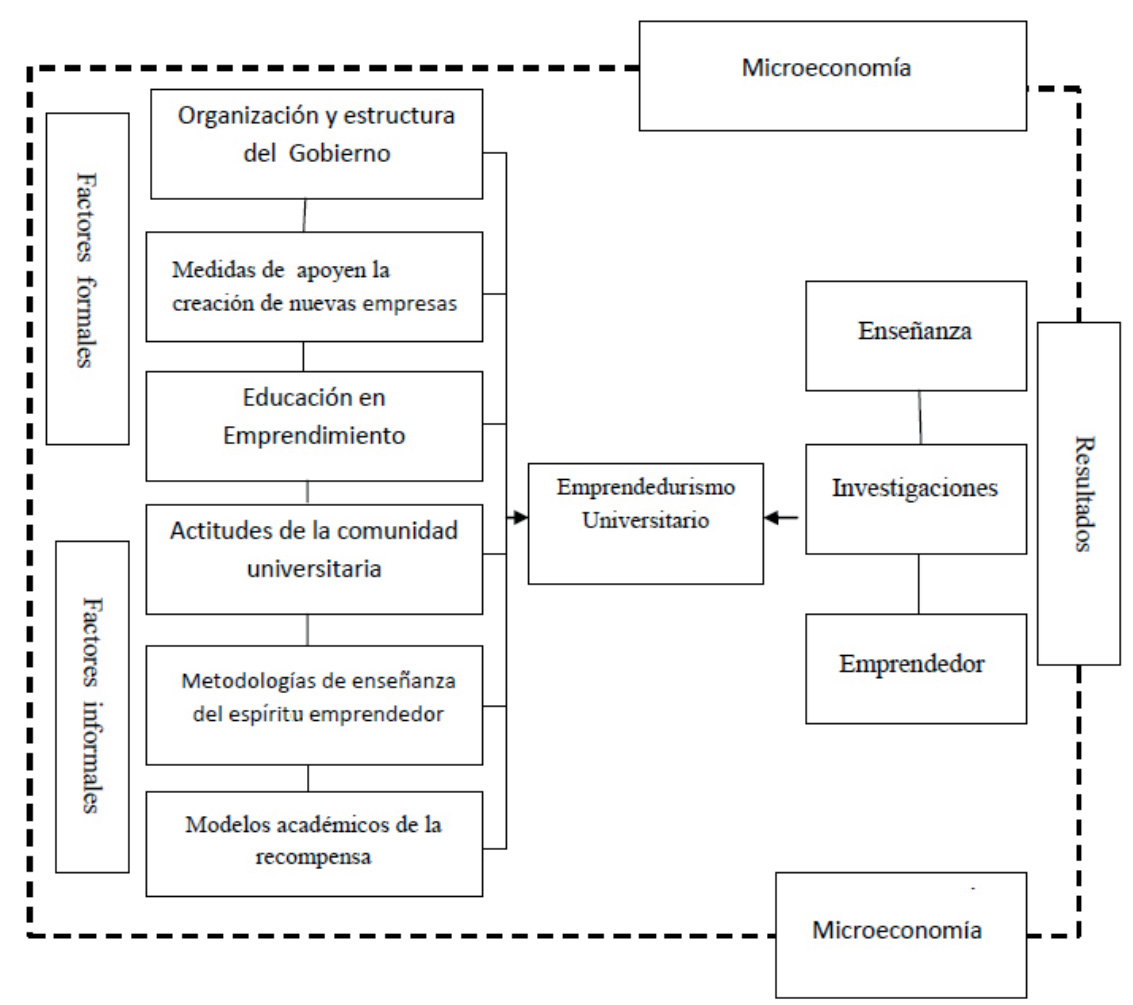

Figura 2. Factores que afectan a la creación y desarrollo de universidades emprendedoras Fuente: (Guerrero, 2006) y (Sooreh, Salamzadeh, \& Safarzadeh, 2011) 
Coello, Goméz y Zuleta (2007), sintetizan la propuesta encaminada a fortalecer la cultura emprendedora, en los miembros de la comunidad universitaria de la Universidad de Oriente, que responda a la necesidad de institucionalizar y de organizar, desde el contexto formal educativo la formación de emprendedores; propiciando al mismo tiempo el desarrollo empresarial local y regional, teniendo como base la premisa, que para el surgimiento de nuevas empresas no es suficiente la motivación y la iniciativa personal, sino que también se requieren conocimientos que permitan la transformación de las ideas de negocio en propuestas sistemáticas, viables y contextualizadas en la realidad socio económica del país y de la región.

La propuesta fundamenta su desarrollo en la implementación, evaluación y seguimiento de cuatro programas entre los cuales se tienen, programa de sensibilización para la adopción de la cultura emprendedora en el ámbito de la comunidad universitaria, mejora de la capacidad de gestión e incorporación del concepto emprendedor en el currículo para el desarrollo de la cultura emprendedora, capacitación para la creación de empresas y creación de la incubadora de empresas en la Universidad, para el desarrollo de la cultura emprendedora, en los cuales se definen objetivos, estrategias, acciones, indicadores, metas, tiempo y los encargados de su ejecución, como uno de los mecanismos que viabiliza la transformación del conocimiento hacia el cambio de visión de los nuevos profesionales, en la búsqueda del desar- rollo y la sustentabilidad, a través de la creación de empresas que produzcan productos, procesos y servicios, satisfagan las necesidades del mercado que a diario cambia, y se vuelve más exigente. También presenta claramente los grupos de interés que se beneficiarán de la puesta en marcha del plan de fortalecimiento de la cultura emprendedora. Estableciendo al mismo tiempo, la importancia de contar con un equipo de docentes de tipo interdisciplinario para la formación de emprendedores, como parte de las fortalezas institucionales, que la puede llevar al posicionamiento a nivel local, regional y nacional, en el contexto de la educación superior universitaria, como una empresa oferente de servicios educativos, con estándares de calidad y acorde a las nuevas tendencias educativas, visualizando en la metodología emprendedora una estrategia de mercadeo buscando la competitividad. En síntesis, el fortalecimiento del concepto emprendedor, se presenta como una alternativa oportuna y estratégica, que permite el abordaje integral de las tres funciones básicas que orientan el ser y el deber ser de toda Institución de Educación Superior.

\section{MÉTODOS}

El presente estudio es descriptivo. La información primaria que se recogió fue a través de la aplicación de encuestas ah doc. La población materia de la presente investigación se basó en docentes y estudiantes de la FCA.

El método de muestreo que sirvió de referencia para la realización de la encuesta es el muestreo probabilístico estratificado, donde

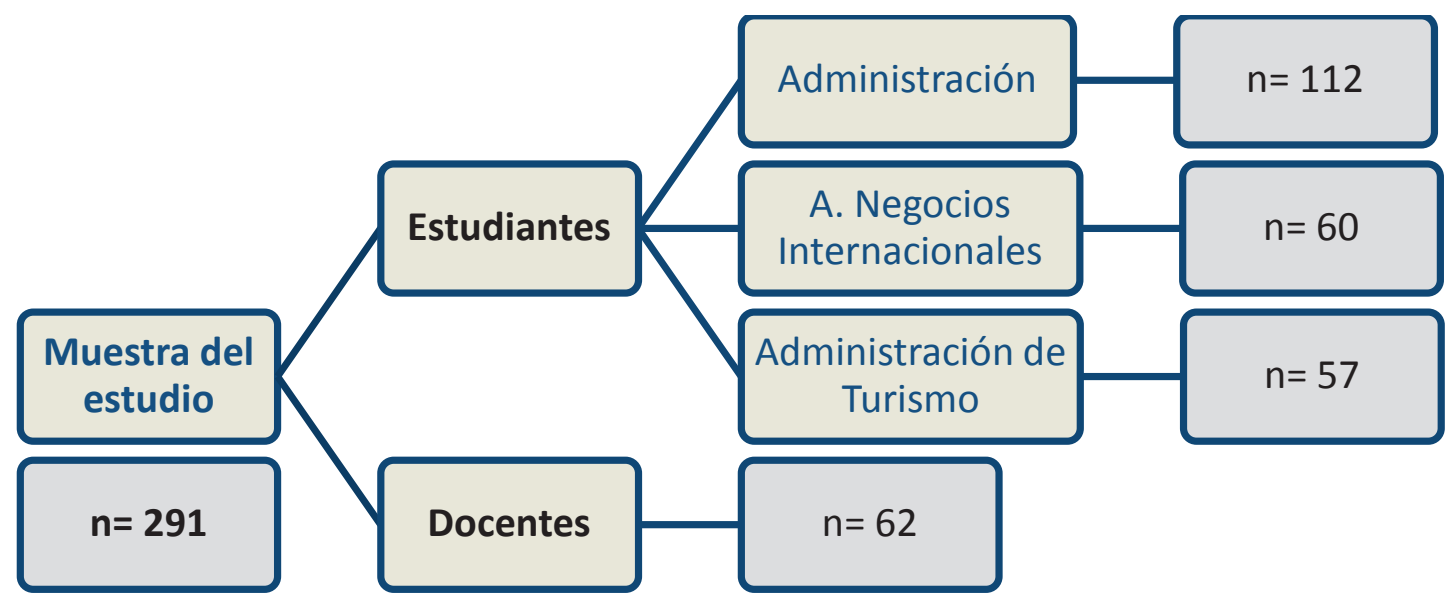

Figura 3. Muestra del estudio la cultura emprendedora en la Facultad de Ciencias Administrativas de la UNMSM. Elaboración: Propia. 
los miembros que pertenecen a un mismo estrato tienen igual probabilidad de ser seleccionados en la muestra en el caso de los estudiantes y para docentes muestreo probabilístico aleatorio simple.

\section{RESULTADOS}

A continuación mostramos y comentamos algunos de los resultados que desde nuestra perspectiva fueron significativos.

\section{A. Principales Resultados de Estudiantes}

1. Considerando que usted estudia en una Facultad ligada a las Ciencias Empresariales, su posición respecto del emprendimiento es:

Tabla 1.

Opinión de emprendimiento, según género

\begin{tabular}{lccc} 
& Masculino & Femenino & Total \\
& $\%$ & $\%$ & $\%$ \\
\hline No quiero tener mi empresa, solo trabajar en el sector público/privado & 10.1 & 3.3 & 6.6 \\
Tengo una idea de negocio desde hace tiempo & 50.5 & 67.5 & 59.4 \\
Estoy juntando capital y socios para iniciar mi empresa & 24.8 & 20.8 & 22.7 \\
Ya inicie mi empresa hace pocos meses & 5.5 & 5.0 & 5.2 \\
Tengo una empresa desde hace más de un año & 9.2 & 3.3 & 6.1 \\
\hline Total & 100.0 & 100.0 & 100.0 \\
\hline
\end{tabular}

Fuente: Elaboración propia

2. ¿Cómo percibe la preparación que realiza la FCA, para que sus alumnos creen su propia empresa?

Tabla 2.

Preparación que realiza la FCA, según Escuela Profesional

\begin{tabular}{lcccc} 
& $\begin{array}{c}\text { Administración } \\
\text { \% }\end{array}$ & $\begin{array}{c}\text { Administración de Negocios } \\
\text { Internacionales }\end{array}$ & $\begin{array}{c}\text { Administración de } \\
\text { Turismo }\end{array}$ & $\begin{array}{c}\text { Total } \\
\%\end{array}$ \\
Nula preparación & 7.1 & 3.3 & $\%$ & $\%$ \\
Poca preparación & 33.0 & 38.3 & 3.5 & 5.2 \\
Regular preparación & 50.9 & 46.7 & 40.4 & 36.2 \\
Buena preparación & 8.9 & 11.7 & 49.1 & 49.3 \\
\hline Total & 100.0 & 100.0 & 7.0 & 9.2 \\
\hline
\end{tabular}

Fuente: Elaboración propia

3. ¿Cómo percibe el ambiente (grupo humano, infraestructura, institución) en la FCA para la promoción del emprendimiento?

Tabla 3.

Percepción del ambiente de la FCA, según EAP

\begin{tabular}{lcccc} 
& Administración & $\begin{array}{c}\text { Administración de Negocios } \\
\text { Internacionales }\end{array}$ & $\begin{array}{c}\text { Administración de } \\
\text { Turismo }\end{array}$ & $\begin{array}{c}\text { Total } \\
\text { Nula }\end{array}$ \\
\% & 6.3 & 10.0 & $\%$ & $\%$ \\
Poca & 32.1 & 28.3 & 3.5 & 6.6 \\
Regular & 42.0 & 40.0 & 36.8 & 32.3 \\
Buena & 19.6 & 20.0 & 47.4 & 42.8 \\
Excelente & 0.0 & 1.7 & 12.3 & 17.9 \\
\hline Total & 100.0 & 100.0 & 0.0 & 0.4 \\
\hline
\end{tabular}

Fuente: Elaboración propia 
4. ¿Cómo calificas las actividades del fomento de cultura emprendedora en la Facultad de Ciencias Administrativas?

Tabla 4.

Calificación de actividades, según EAP del estudiante FCA

\begin{tabular}{lcccc} 
& Administración & $\begin{array}{c}\text { Administración de Negocios } \\
\text { Internacionales }\end{array}$ & $\begin{array}{c}\text { Administración de } \\
\text { Turismo }\end{array}$ & Total \\
\hline Nulo fomento & $\%$ & $\%$ & $\%$ & $\%$ \\
Poco fomento & 3.60 & 1.70 & 0.00 & 2.20 \\
Regular fomento & 30.40 & 28.30 & 35.10 & 31.00 \\
Buen fomento & 53.60 & 51.70 & 54.40 & 53.30 \\
Excelente fomento & 10.70 & 18.30 & 10.50 & 12.70 \\
\hline Total & 1.80 & 0.00 & 0.00 & 0.90 \\
\hline
\end{tabular}

Fuente: Elaboración propia

\section{B. Principales Resultados de la Encuesta a Docentes}

5. Según su conocimiento del plan de estudio de la(s) escuela(s) donde usted enseña, ¿Considera que motiva al estudiante a ser emprendedor?

Tabla 5.

Nivel de motivación del Plan de Estudios a ser emprendedor, según género del docente

\begin{tabular}{lccc} 
& Masculino & Femenino & Total \\
& $\%$ & $\%$ & $\%$ \\
El plan de estudio no motiva & 3.8 & 11.1 & 4.8 \\
El plan de estudio motiva escasamente & 43.4 & 22.2 & 40.3 \\
El plan de estudio motiva en forma regular & 28.3 & 55.6 & 32.3 \\
El plan de estudio tiene buena motivación & 22.6 & 11.1 & 21.0 \\
El plan de estudio motiva excelentemente & 1.9 & 0.0 & 1.6 \\
\hline Total & 100.0 & 100.0 & 100.0 \\
\hline
\end{tabular}

Fuente: Elaboración propia

6. Le parece que es suficiente los diferentes concursos y ferias desarrolladas por parte la facultad para el desarrollo de emprendedores.

Tabla 6.

Concursos y ferias en la FCA para la promoción del emprendimiento en los estudiantes, según EP

\begin{tabular}{lcccc} 
& Administración & $\begin{array}{c}\text { Administración de Negocios } \\
\text { Internacionales }\end{array}$ & $\begin{array}{c}\text { Administración } \\
\text { de Turismo }\end{array}$ & $\begin{array}{c}\text { Total } \\
\text { Es Insuficiente }\end{array}$ \\
Es escasamente suficiente & 5.0 & $\%$ & $\%$ & $\mathbf{N}^{\circ}$ \\
Es regularmente suficiente & 52.5 & 9.1 & 9.1 & 4 \\
Es suficiente & 25.0 & 27.3 & 54.5 & 30 \\
Es muy suficiente & 15.0 & 9.1 & 27.3 & 14 \\
\hline Total & 2.5 & 45.5 & 9.1 & 12 \\
\hline
\end{tabular}

Fuente: Elaboración propia 
7. ¿Cómo califica las actividades del fomento de la cultura emprendedora en la FCA?

Tabla 7.

Calificación de actividades de la cultura emprendedora, según EP

\begin{tabular}{|c|c|c|c|c|}
\hline & Administración & $\begin{array}{l}\text { Administración de Negocios } \\
\text { Internacionales }\end{array}$ & $\begin{array}{c}\text { Administración de } \\
\text { Turismo }\end{array}$ & Total \\
\hline & $\%$ & $\%$ & $\%$ & $\mathbf{N}^{\circ}$ \\
\hline Nulo fomento & 7.5 & 27.3 & 0.0 & 9.7 \\
\hline Poco fomento & 47.5 & 27.3 & 81.8 & 50.0 \\
\hline Regular fomento & 32.5 & 27.3 & 9.1 & 27.4 \\
\hline Buen fomento & 12.5 & 18.2 & 0.0 & 11.3 \\
\hline Excelente fomento & 0.0 & 0.0 & 9.1 & 1.6 \\
\hline Total & 100.0 & 100.0 & 100.0 & 100.0 \\
\hline
\end{tabular}

Fuente: Elaboración propia

8. ¿Considera usted que la FCA debería intensificar la formación de estudiantes emprendedores?

Tabla 8.

Opinión del docente con respecto a la intensificación en la formación de estudiantes emprendedores, según EP

\begin{tabular}{lcccc} 
& $\begin{array}{c}\text { Administración } \\
\text { \% }\end{array}$ & $\begin{array}{c}\text { Administración de Negocios } \\
\text { Internacionales }\end{array}$ & $\begin{array}{c}\text { Administración de } \\
\text { Turismo }\end{array}$ & Total \\
Nulo & 0.0 & $\%$ & $\%$ & $\%$ \\
Poco & 10.0 & 0.0 & 0.0 & 0.0 \\
Mucho & 90.0 & 27.3 & 9.1 & 12.9 \\
\hline Total & 100.0 & 72.7 & 90.9 & 87.1 \\
\hline
\end{tabular}

Fuente: Elaboración propia

9. ¿En qué grado usted conoce acerca de los proyectos, convenios y áreas de emprendimiento que tiene la FCA?

Tabla 9.

Conocimiento de convenios vigentes por los profesores, según EP

\begin{tabular}{lcccc} 
& Administración & $\begin{array}{c}\text { Administración de Negocios } \\
\text { Internacionales }\end{array}$ & $\begin{array}{c}\text { Administración de } \\
\text { Turismo }\end{array}$ & Total \\
\hline Desconozco totalmente & $\%$ & $\%$ & $\%$ & $\%$ \\
Conozco escasamente & 25.0 & 45.5 & 45.5 & 32.3 \\
Conozco regularmente & 35.0 & 18.2 & 18.2 & 16.1 \\
Conozco suficientemente & 27.5 & 27.3 & 27.3 & 29.0 \\
Conozco totalmente & 2.5 & 9.1 & 9.1 & 21.0 \\
\hline Total & 100.0 & 0.0 & 0.0 & 1.6 \\
\hline
\end{tabular}

Fuente: Elaboración propia 


\section{Principales Resultados de la aplicación de la prueba Chi Cuadrado}

\section{C.1 Encuesta Aplicada a los Estudiantes}

\section{Genero vs Opinión de emprendimiento}

Ho: El género del estudiante y la opinión de emprendimiento son independientes.

H1: El género del estudiante y la opinión de emprendimiento están relacionados.

$$
\text { Total M: } 109 \text { F: } 120 \quad n=229
$$

Chi-cuadrada $=10.381, G L=4$, Valor $P=0.034$ asumiendo una significancia del $5 \%$

El p valor $<0.05$ indica que se rechaza Ho y se acepta $\mathrm{H} 1$

2. Proporciones Múltiples: Percepción de los estudiantes para el emprendimiento según Escuela Profesional

Ho: La percepción respecto a la preparación para el emprendimiento en las tres escuelas profesional es la misma.

H1: La percepción respecto a la preparación para el emprendimiento en las tres escuelas profesional es diferente.

Total ADM: 112 NN.II: 60 TURISM: 57 n=229

Chi-cuadrada $=0.969, G L=4$, Valor $P=0.914$

El p valor $>0.05$ indica que se acepta Ho

\section{C.2 Encuesta Aplicada a los docentes}

3. Proporciones Múltiples: Percepción de los docentes sobre las actividades de cultura emprendedora

Ho: La percepción de los docentes respecto a las actividades de la cultura emprendedora es la misma en las tres escuelas profesionales.

H1: La percepción de los docentes respecto a las actividades de la cultura emprendedora es diferente en las tres escuelas profesionales.

Total ADM: $38 \quad$ NN.II-TURISM: $22 \quad N=60$

Chi-cuadrada $=0.624, G L=1$, Valor $P=0.430$

El p valor $>0.05$ indica que se acepta Ho

\section{Análisis De Los Resultados De La In- vestigación}

La cobertura de la encuesta fue dirigida a los estudiantes y docentes de las tres (03) escuelas profesionales de la Facultad de Ciencias Administrativas de la UNMSM.

El tamaño de la muestra estudiantes fue Escuela de Administración (112), seguido por la escuela Administración de Negocios Internacionales (60) y Administración de Turismo (57). En tanto que en los docentes se aplicó 60 encuestas. No se pudo disgregar como en los alumnos, dado que muchos docentes dictan cursos en más de una escuela.

\section{Estudiantes:}

- Los estudiantes en su mayoría (59\%) concluyen que solo "tienen una idea de negocio", lo cual demuestra una incipiente iniciativa de negocio, pero que en el proceso formativo, pueden modificar favorablemente para emprender efectivamente un negocio propio. Sin embargo, hay un $34 \%$ de los estudiantes que ya tienen claro en su idea de emprendimiento empresarial e incluso ya vienen desarrollando. Saber de la existencia de este grupo de estudiantes es alentador, dado que ellos representan un segmento donde la teoría se lleva a la práctica.

- Los estudiantes perciben que la FCA les brinda una "regular y buena preparación" para que formen sus propias empresas en un $58.5 \%$, esto se complementa con la percepción respecto al ambiente (grupo humano, infraestructura, institución) para la promoción del emprendimiento que también lo califican como "regular ambiente" en un $43 \%$ y "buena" en un $18 \%$. Tales resultados evidencian que el proceso de preparación, así como la infraestructura están siendo valorados en forma positiva y constructiva por la mayoría de los estudiantes. Obviamente no es lo mejor, pero las percepciones en ambos criterios son aceptables.

- En relación al fomento del emprendimiento, los estudiantes encuestados 
manifiestan como regular en un 53.30\% y buena en un $12.70 \%$. E incluso hay el $1 \%$ dicen que el fomento al emprendimiento es excelente. A nivel de escuelas los estudiantes de Negocios Internacionales perciben mejor, dado que el $60 \%$ se ubican entre regular y buena, seguido de estudiantes de Administración de Turismo con un $65 \%$ y Administración con $64 \%$, respectivamente.

- De la aplicación de la prueba Chi cuadrado se observa que existe una relación entre el género del estudiante y la opinión de emprendimiento. Las Estudiantes mujeres tienes mayores opiniones emprendedoras, en relación a sus compañeros varones.

\section{Docentes}

- La opinión de los docentes respecto al plan de estudio de la escuela profesional donde enseñan, la consideran como que "motivan en forma regular" con un $32.3 \%$, en tanto el $22.6 \%$ consideran como que el plan de estudios tiene buena y excelente. A nivel de género son las docentes damas las que tienen un mejor percepción del plan de estudios con un $66.7 \%$, en tanto a nivel de docentes hombres representan el 50.9\%.

- A nivel de realización de actividades para la promoción de la cultura emprendedora, el $50 \%$ de los encuestados consideran que hay poco fomento, en tanto que el $27 \%$ dicen regular fomento, $13 \%$ consideran que hay buen y excelente fomento.

- Sobre el nivel de intensificación en el proceso formativo en la formación de estudiantes emprendedores, hay una plena coincidencia de la mayoría absoluta. Así el $87 \%$ considera que se debe intensificar mucho, y el 13\% restante manifiestan que se debe intensificar poco. Esta percepción de la necesidad de intensificar se visualiza en las escuelas de Administración y Administración de Turismo.

- Sobre el nivel de conocimiento de los proyectos, convenios y otras acciones vi- gentes en la universidad, la mayoría de los encuestados manifestaron desconocerlas. Prácticamente el 78 de los encuestados manifestaron desconocer o conocer muy poco. Solo el $22 \%$ de la muestra dijeron conocer suficientemente.

- De la misma forma que los estudiantes, no hay una diferencia significativa entre la percepción de los docentes, respecto a las actividades del cultura emprendedora.

\section{DISCUSIÓN}

A través de los autores y teorías revisadas, podemos identificar los factores que estimulan el espíritu emprendedor, y estas se relacionan fundamentalmente a dos elementos: la educación y el entorno donde se desenvuelven los emprendedores. En ese orden de ideas, la Universidad es un espacio por excelencia para formar y/o modular las competencias de los estudiantes en conductas y actitudes emprendedoras. Así la Facultad de Ciencias Administrativas, en líneas generales goza de un nivel de percepción aceptable, de parte de los estudiantes y docentes para promover la cultura emprendedora.

Tal como postulan los autores revisadas para remozar la teoría, los resultados de la investigación, corroboran el papel relevante de la Universidad en la generación de conductas y actitudes emprendedoras. La Universidad es un espacio para incorporar conocimientos nuevos, remozar los vigentes. Es un laboratorio donde germinan nuevas ideas. Ideas que nacen e ideas que al ponerse en práctica, algunas revolucionan y florecen, otras fracasan, pero sirven como experiencia de aprendizaje en los alumnos. Los resultados de esta investigación nos sirven para retroalimentar en la reestructuración de los planes y proyectos curriculares. El empoderamiento de una cultura emprendedora pasa necesariamente porque los docentes diseñen en el proceso formativo, planes de ideas de negocios, promuevan a través de capitales semillas, la puesta en práctica de esas ideas, asesoren con sus conocimientos y experiencia, para que los alumnos se sientan apoyados y fortalecidos para perseverar en sus proyectos de emprendimiento.

Creemos que estas son las tareas inmediatas, que se deben ponerse en práctica, si se de- 
sea cambiar el perfil de una Universidad libresca, a una Universidad emprendedora. Son estos factores que no están siendo fortalecidos en la FCA de la UNMSM, que como vemos son claves para mejorar la cultura emprendedora.

\section{CONCLUSIONES}

- Se evidencia una cultura emprendedora media en la FCA por parte de docentes $\mathrm{y}$ alumnos, pero ambos manifiestan la necesidad de intensificar el fomento de la cultura emprendedora.

- Existe la necesidad de un modelo integrado de gestión y enseñanza que implique a docentes, estudiantes y la FCA como institución para incrementar y fortalecer la cultura emprendedora, esto a través de la reestructuración de mallas curriculares acorde a un sistema que fomente dicha cultura y complementarlo con talleres, ferias y concursos que fortalezcan. Tales acciones va mejorar la percepción actual de los estudiantes.

- El sistema de comunicación actual para docentes y alumnos de las iniciativas realizadas por parte de la facultad en emprendimiento no son del todo eficaz porque son esfuerzos aislados donde la mayoría desconocen de ellas. Se hace necesario que desde el decanato se instituya una oficina que promueva, apoye e incluso busque mercados para que las ideas de emprendimiento cuaje y se desarrolle. Como en el caso de la Micro y Pequeñas Empresas, hay políticas de promoción y fomento desde el Gobierno Central, de la misma forma se hace necesario que la Universidad promueva con financiamientos iniciales a modo de capitales semilla, las iniciativas de emprendimiento entre sus estudiantes.

- Finalmente complementario a estas medidas es necesario la implementación de una mejor infraestructura, incremento de capacitaciones del grupo humano y elaboración de un plan como institución que consideren el fomento de la cultura emprendedora un pilar base de la formación que se brinda esto integrado al plan estratégico.

\section{AGRADECIMIENTOS}

Agradecemos a nuestros estudiantes de la Facultad de Ciencias Administrativas, que son la razón de nuestros esfuerzos como docentes e investigadores, y a los colegas que nos permitieron recopilar sus percepciones para la elaboración del presente trabajo de investigación.

\section{REFERENCIAS BIBLIOGRÁFICAS}

Campos, A., Hormiga, E., \& Valls, J. (2014). El fomento de la cultura emprendedora y la mejora de la formación en emprendimiento e innovación. Barcelona: Red Emprendia.

Eda, C. L., \& Cristina, C. L. (2007). Cultura e atitude empreendedora: um estudo em empresas no distrito federal. Faces Journal, 90-100.

Eoi, W. (15 de Marzo de 2017). EOI. Obtenido de http://www.eoi.es/wiki/index.php/Cultura emprendedora_en_Proyectos_de_negocio.

Guerrero, M. (2006). A literature review on entrepreneurial universities: an institutional approach. Autonomous University of Barcelona.Barcelona.: Business Economic Department, Autonomous.

Narváez, M. (2012). Dimensiones del emprendedurismo desde una vision universitaria. ING-NOVACIÓN ,(2)4, 1-7.

Perez, E., Sumari, J., Quintana, G., \& Uyehara, J. (2013). Cuantificación del potencial emprendedor de los alumnos de la facultad de Ciencias Administrativas de la UNMSM. Gestión del Tercer Milenio , 37-46.

Sooreh, L., Salamzadeh, A., \& Safarzadeh, H. (2011). Defining andMeasuring Entrepreneurial Universities: A Study in Iranian Context Using Importance-Performance Analysis and TOPSIS Technique. Global Business and Management Research: An International Journal , 182-199. 